\title{
WNT5A expression is regulated by the status of its promoter methylation in leukaemia and can inhibit leukemic cell malignant proliferation
}

\author{
GANG DENG ${ }^{1}$, ZHAO QUAN LI ${ }^{1}$, CHEN ZHAO $^{1}$, YUAN YUAN ${ }^{1}$, \\ CHANG CHUN NIU ${ }^{1}, \mathrm{CHEN} \mathrm{ZHAO}^{2}$, JING PAN ${ }^{1}$ and WEI KE SI ${ }^{1}$ \\ ${ }^{1}$ Department of Clinical Hematology, ${ }^{2}$ Twelve Class, Student Brigade, \\ Third Military Medical University, Chongqing 400038, P.R. China
}

Received August 12, 2010; Accepted October 14, 2010

DOI: $10.3892 /$ or.2010.1108

\begin{abstract}
Although down-regulation of WNT5A expression has been reported in some types of leukaemias, the level of WNT5A expression has not been assessed in leukaemia complete remission (CR) cases, the relationship among WNT5A expression level, the status of its promoter methylation, and the curative effect of leukaemia has not been reported, and the effect of WNT5A on cell proliferation has not been assessed. In this study, we analyzed WNT5A expression in various kinds of leukaemia cases, leukaemia CR cases, non-malignant hematopoietic ( $\mathrm{NMH}$ ) cases, as well as in leukemic cell lines and $\mathrm{CD} 34^{+}$cells. The methylation status of the WNT5A promoter and the levels of the Wnt5a protein were also studied. We also investigated the effect of Wnt5a on leukemic cell proliferation. WNT5A expression level was higher in NMH but lower in leukaemia cases compared to that in $\mathrm{CR}$-cases $(\mathrm{P}<0.01)$, and was expressed at low level in leukemic cell lines K562, U937 and Jurkat. Wnt5a protein was positive in $\mathrm{NMH}, \mathrm{CR}$ cases and $\mathrm{CD} 34^{+}$, but negative in leukaemia cases. WNT5A promoter was methylated in leukaemia cases and all leukemic cell lines, but not in NMH and CR cases. WNT5A expression was up-regulated after exposure to the demethylating agent 5Aza-2'-deoxycytidine (Aza) in the K562, U937, Jurkat leukemic cell lines and in $83.3 \%$ (10/12) of CR patients after cure, respectively. The increased Wnt5a protein can inhibit K562 malignant proliferation and arrest cell cycle at the G2/M phase after exposure to Aza. These results indicate that WNT5A expression was restored in complete remission cases due to demethylation, and Wnt5a can
\end{abstract}

Correspondence to: Dr Wei Ke Si, Department of Clinical Hematology, Third Military Medical University, Chongqing 400038, P.R. China

E-mail: weikesi2004@hotmail.com

Key words: WNT5A, leukaemia, methylation, complete remission cases inhibit leukaemic cell proliferation. We propose that WNT5A can act as a suppressor factor in leukemogenesis and can be used as a potential marker for curative effect assessment in leukaemia.

\section{Introduction}

Wnt proteins are a large secreted glycoprotein family that play important roles in mediating vital biological processes like embryogenesis, organogenesis and tumorigenesis (1-4). Wnt signaling has been divided into the canonical and noncanonical pathways. In the canonical pathway, Wnt binds to the Frizzle receptor leading to the inhibition of a multiple protein complex that otherwise can phosphorylate and degrade $\beta$-catenin. The stabilized $\beta$-catenin can thus translocate into nucleus and be responsible for the activation of gene transcription of $\mathrm{Wnt} / \mathrm{ß}$-catenin target genes including cyclin D1 and c-myc (5-8). Wnt5a, in the presence of specific FZ4, could also activate the canonical Wnt signaling pathway, and the overexpression of Wnt5a has ample opportunity to promote cell proliferation because of the up-regulation of cyclin D1 and c-myc. The non-canonical signaling pathway, although not completely understood, is independent of Bcatenin and mainly includes three defined pathways. The planar cell polarity: Vertebrate Wnt5a and Wnt11 initiate the signaling via Fz, which can operate through the cytoskeleton to control cell orientation and movement. This ability of Wnt5a to promote cell movement has crucial implications for normal development or cancer progression (9). The $\mathrm{Wnt} / \mathrm{Ca}^{2+}$ pathways: Wnt5a and Fz2 may regulate intracellular $\mathrm{Ca}^{2+}$ flux and levels which can lead to the activation of calmodulin-dependent protein Kinase II (CAMKII) and protein kinase $\mathrm{C}$ (PKC) (10). This pathway also can stimulate the TAK1-NLK pathway to phosphorylate (11), inactivate the active $\beta$-catenin transcription complex and inhibit the activation of the canonical signaling $(12,13)$. The ROR2 pathways: Wnt5a can bind and activate the ROR2 tyrosine kinase receptor resulting in the activation of the actin-binding protein, filamin A, and the JNK signaling pathway $(11,14)$. This pathway also can stimulate the TAK1NLK pathway to phosphorylate (11) and inactivate the active ß-catenin transcription complex $(12,15)$. Wnt5a is one of 
the most highly investigated non-canonical Wnts, and has been shown to be involved in almost all aspects of the non-canonical Wnt signaling pathway. It has been proposed that Wnt5a may have oncogenic function. Up-regulation of WNT5A has been observed in human primary breast cancer, gastric cancer, lung cancer, and prostate cancer (16-19), and Wnt5a has been reported to facilitate cell invasion in human metastatic melanoma (20). However, the function of Wnt5a is complicated as recent findings suggested that it may have an anti-oncogenic role. Decreased expression of WNT5A was observed in endometrial carcinoma and thyroid carcinoma $(21,22)$. Wnt5a can activate different Wnt signaling pathways, depending on which receptors are present at the cell surface $(13,15)$. It may have a tumour suppressing or an oncogenic effect depending on the receptors in different cancer types. Hence, the presence or absence of these receptors may, to some extent, explain the conflicting role of WNT5A in different cancers. WNT5A also has tumor suppressor activity in hematopoietic tissue (23). WNT5A is epigenetically silenced in hematologic malignancies, such as ALL, by aberrant methylation (24). WNT5A down-regulation or silencing is also found in multiple hematologic tumor cell lines including nasal NK/T-cell lymphoma and NK leukaemia cell lines, leukaemia and Burkitt lymphoma (25). However, the relationship between the expression level of WNT5A and the methylation status of its promoter in various kinds of leukaemia cases is not completely clear, especially in the leukaemia complete remission (CR) cases. Here, we analyzed WNT5A expression in bone marrow of complete remission cases (CR), leukaemia cases and non-malignant hematopoietic cases (as hyperplasia diseases of bone marrow), and analyzed WNT5A expression in $\mathrm{CD}_{3} 4^{+}$cells from umbilical cord blood (mainly including hematopoietic progenitor cells, a population of non-malignant proliferation cells) and leukemic cell lines (malignant proliferation cells), analysed the methylation status of the WNT5A promoter in leukemic cell lines, leukaemia cases, CR cases, and observed the effect of Wnt5a on leukemic cell line K652 proliferation after treatment with the Aza.

In this study, we demonstrated that WNT5A expression was down-regulated in leukaemia due to its promoter aberrant methylation but restored in complete remission cases owing to demethylating, and Wnt5a inhibited leukemic cell malignant proliferation. We propose that Wnt5a plays an important role in inhibiting leukemogenesis.

\section{Materials and methods}

Cell lines. Three leukaemia-derived cell lines (K562, U937, Jurkat) were preserved in our laboratory. Cells were grown at $37^{\circ} \mathrm{C}$ under $5 \% \mathrm{CO}_{2}$ in humidified air in an RPMI medium (Gibco, Invitrogen Corp., USA) supplemented with $20 \%$ foetal bovine serum (HyClone, USA), $1 \%$ penicillin/streptomycin and $1 \%$ HEPES (Gibco).

Human subjects. We studied 68 leukaemia patients (37 male, 31 female), 18 ALL, 12 CLL, 10 NHL, 16 AML, 12 CML and 35 completely remission (CR) patients (23 male, 12 female), 8 ALL-CR, 5 CLL-CR, 6 NHL-CR 12, AML-CR, 4 CML-CR, who were diagnosed between August 2008 and March 2010
Table I. Main clinical characteristics of the patients and the outcome of WNT5A expression.

\begin{tabular}{|c|c|c|}
\hline Feature & $\begin{array}{c}\text { Negative WNT5A } \\
\text { expression } \\
\text { (no. of patients) }\end{array}$ & $\begin{array}{c}\text { Positive WNT5A } \\
\text { expression } \\
\text { (no. of patients) }\end{array}$ \\
\hline \multicolumn{3}{|l|}{ Age (years) } \\
\hline$\leq 15$ & 22 & 18 \\
\hline$>15$ & 51 & 12 \\
\hline \multicolumn{3}{|l|}{ Gender } \\
\hline Male & 38 & 22 \\
\hline Female & 23 & 20 \\
\hline \multicolumn{3}{|l|}{ WBC count } \\
\hline$\leq 50 \times 10^{9} / 1$ & 18 & 20 \\
\hline$>50 \times 10^{9} / 1$ & 55 & 10 \\
\hline \multicolumn{3}{|c|}{ FAB classification } \\
\hline M1 & 1 & 3 \\
\hline M2 & 10 & 4 \\
\hline M3 & 3 & 0 \\
\hline M4 & 2 & 0 \\
\hline M5 & 3 & 1 \\
\hline M6 & 2 & 0 \\
\hline CML & 19 & 0 \\
\hline ALL & 11 & 0 \\
\hline NHL & 5 & 1 \\
\hline CLL & 2 & 1 \\
\hline \multicolumn{3}{|l|}{ CR cases } \\
\hline M2 & 5 & 3 \\
\hline M3 & 0 & 4 \\
\hline M4 & 1 & 0 \\
\hline M5 & 1 & 3 \\
\hline M6 & 0 & 1 \\
\hline CML & 2 & 4 \\
\hline ALL & 2 & 5 \\
\hline NHL & 1 & 3 \\
\hline
\end{tabular}

Negative WNT5A expression, no gel electrophoretic band in the RT-PCR assay. Positive WNT5A expression, visible gel electrophoretic bands in the RT-PCR assay.

at the Xin-Qiao Hospital and South-West Hospital of the Third Military Medical University China. The median age at diagnosis was 35 year (range 8-72). Patients were studied at the time of initial diagnosis. We also studied the 27 nonmalignant hematopoietic cases (14 male, 13 female) as hyperplasia diseases [7 iron deficiency anemia (IDA)], 7 hemolytic anemia (HA), 3 idiopathic thrombocytopenic purpura (ITP), 3 essential thrombocythemia (ET), 2 polycythemia vera (PV), 5 leukemoid reaction (LR). The median age at diagnosis was 39.5 years old (range 10-69) and the $\mathrm{CD}_{34}{ }^{+}$cells from 10 case umbilical cord blood of normal births. Informed consent was obtained from the patients or the patient's guardians. Diagnosis was established according to standard morphologic, cytochemical and immuno- 
phenotypic criteria. Clinical characteristics of the patients are listed in Table I.

RNA extraction and RT-PCR. Bone marrow mononuclear cells were lysed with cell lysis solution (139.6 mmol/1 chlorine ammonia, $16.96 \mathrm{mM}$, Tris-buffer). Total RNA was isolated using TRIzol reagent (Invitrogen) and cDNA was synthesized from RNA by M-MLV reverse transcriptase (Toyobo, Japan) with oligo-dT primers (Sango Technology, China). The primer sequences of WNT5A genes were as follows: WNT5A upstream: 5'-TGTGGTTTAATGGTGCC TGA-3', WNT5A downstream: 5'-TTCGTCGTGCTCAAG GTATG-3'. GAPDH upstream: 5'-CATCACCATCTTCC AGGAGCG-3', GAPDH downstream: 5'-TGACCTTGCCC ACAGCCTTG-3'. The reaction mixture was first denatured at $94^{\circ} \mathrm{C}$ for $5 \mathrm{~min}$. The PCR conditions were as follow: $94^{\circ} \mathrm{C}$ for $45 \mathrm{sec}, 58^{\circ} \mathrm{C}$ for $35 \mathrm{sec}$ and $72^{\circ} \mathrm{C}$ for $45 \mathrm{sec}$ for 40 cycles (for WNT5A) or 28 cycles (for $G A P D H$ ), followed by $72^{\circ} \mathrm{C}$ for $7 \mathrm{~min}$. PCR products were visualized by ethidium bromide staining after agarose gel electrophoresis at sizes of $253 \mathrm{bp}(W N T 5 A)$ and $443 \mathrm{bp}(G A P D H)$, respectively. Expression of $W N T 5 A$ was presented by the relative yield of the PCR products of the target sequence to that of the GAPDH gene. The semi-quantitative Formula is INT* $\mathrm{mm}^{2}$ (WNT5A)/INT* $\mathrm{mm}^{2}(G A P D H)$.

Treatment with the demethylating agent 5-Aza-2'-deoxycytidine. Leukaemia-derived cell lines K562, U937, Jurkat were grown at a density of $1 \times 10^{6}$ cells $/ \mathrm{ml}$ in $100 \mathrm{ml}$ flasks with $10 \mathrm{ml}$ of RPMI-1640 medium supplemented with $20 \%$ fetal bovine serum and maintained at $37^{\circ} \mathrm{C}$ in a humid atmosphere containing $5 \% \mathrm{CO}_{2}$. Cell lines were treated with $4 \mu \mathrm{M}$ Aza (Sigma-Aldrich, Steinheim, Germany) for 2 days for methylation-specific assays, and the untreated cell lines were regarded as a control. After treatment, cells were washed in PBS, pelleted by centrifugation at $1500 \mathrm{rpm}$ for $5 \mathrm{~min}$ and used for genomic DNA and RNA isolation. DNA was extracted using Tiangen DNA Mini Kit (Tiangen, China). One microgram of total RNA was used for cDNA synthesis by M-MLV reverse transcriptase (Toyobo, Japan) with oligodT primers (Sango Technology, China).

Methylation-specific PCR (MSP). Analysis of the WNT5A (GeneBank: NC_000003) has revealed that WNT5A possesses a 229 bp CpG island located between nt47 and nt225, showing $\mathrm{C}+\mathrm{G}$ content $>60 \%$ and frequency of $\mathrm{CpG}$ island $>0.6$. Methylation status of the $\mathrm{CpG}$ islands in the WNT5A gene promoter was performed by genomic DNA bisulphite treatment followed by MSP. The primers for WNT5A-MD (M): 5'-GTATTTTTCGGAGAAAAAGTTATGC-3', WNT5A-MR: 5'-ACAACCGCGAATTAATATAAACG-3'. The primers for the unmethylated (U), WNT5A-UD: 5'-GGTATTTTTTGGAG AAAAAGTTATGTG-3', WNT5A-UR: 5'-CTACAACCAC AAATTAATATAAACATC-3'. PCR was carried out for 30 cycles consisting of denaturation at $95^{\circ} \mathrm{C}$ for $1 \mathrm{~min}$, annealing at $60^{\circ} \mathrm{C}$ for $1 \mathrm{~min}$, and extension at $72^{\circ} \mathrm{C}$ for $1 \mathrm{~min}$, followed by a final $7 \mathrm{~min}$ extension for all primer sets. The products were separated by electrophoresis on a $2 \%$ agarose gel. Results were always confirmed by repeat MSP assays after an independently performed bisulfite treatment.
Immunocytochemical staining. Cells were fixed on slide in polyformaldehyde for $30 \mathrm{~min}$ at $4^{\circ} \mathrm{C}$, rinsed and blocked for $1 \mathrm{~h}$ in $5 \%$ fetal bovine serum at room temperature. The cells were incubated with anti-Wnt5a polyclonal antibody $(1: 100$ to $1: 500$, Santa Cruz Biotechnology) at $4^{\circ} \mathrm{C}$ overnight or $37^{\circ} \mathrm{C}$ for $60 \mathrm{~min}$. After washing in PBS, the cells were incubated with a biotin-labeled secondary antibody (1:2000 to $1: 10000$, Sigma) for $1 \mathrm{~h}$ at room temperature, and DAB mixture was added and incubated for $20 \mathrm{~min}$ at room temperature. Normal rabbit serum in replace of primary antiserum is used as negative control. The slides were counterstained with hematoxylin.

MTT assay. Cell viability of the K562, U937, Jurkat was measured by the MTT (3-[4,5-dimethylthiazol-2-yl]-2,5diphenyltetrazolium bromide) (Sigma Amresco, USA) before and after the treatment with Aza at the 24, 48, 72, $96 \mathrm{~h}$. MTT was prepared as $5 \mathrm{mg} / \mathrm{ml}$ in phosphate-buffer solution at $37^{\circ} \mathrm{C}$ just before use. A total of $900 \mu 1 \mathrm{MTT}$ solution was added to each well and incubated at $37^{\circ} \mathrm{C}$ temperature and $5 \% \mathrm{CO}_{2}$ and $95 \%$ relative humidity for $4 \mathrm{~h}$. After incubation, MTT was aspirated and $250 \mu \mathrm{l}$ per well of acidic solution of isopropanol was added to each well to dissolve the formazan precipitate. Subsequently, ELISA Reader (Bio-Rad Model 680 , USA) read the optical densities of the plates at $570 \mathrm{~nm}$.

Western blot assay. After the K562, U937 and Jurkat was treated with Aza for $48 \mathrm{~h}$, cells were washed with ice-cold PBS twice and lysed on ice with lysis buffer (Sigma, USA). The insoluble material was removed by centrifugation at $4^{\circ} \mathrm{C}$ (14000 x g for $10 \mathrm{~min}$ ). Equal amounts of proteins were run on $10 \%$ SDS-PAGE gels and transferred to nitrocellulose membrane. After blocking with 5\% skim milk, the membranes were subjected to immunoblotting with appropriate primary antibody (mouse monoclonal antibody Wnt5a IgG or Actin IgG) overnight at $4^{\circ} \mathrm{C}$. After washing and further incubation with secondary antibody (horseradish peroxidaseconjugated goat anti-mouse $\mathrm{IgG}$ ) at $37^{\circ} \mathrm{C}$ for $60 \mathrm{~min}$, bands were visualized using an enhanced chemiluminescence system (Thermo Scientific, USA). Densitometric analysis of Western blots was carried out using Gel-Pro system (Bio-Rad, USA).

Flow cytometric analysis of cell cycle. After 48-h treatment with Aza $(4 \mu \mathrm{M})$ cells were harvested, washed in cold PBS, fixed in $70 \%$ ethanol and stored at $4^{\circ} \mathrm{C}$. [Cells may be stored in $70 \%$ ethanol at $-20^{\circ} \mathrm{C}$ for several weeks prior to propidium iodide (PI) staining and flow cytometric analysis]. Cells were washed in PBS, $1 \mathrm{ml} \mathrm{PI}(50 \mu \mathrm{g} / \mathrm{ml})$ of staining solution was added to the cell pellet and mixed well, then $50 \mu \mathrm{l}$ of RNase A stock solution was added, followed by incubation for $3 \mathrm{~h}$ at $4^{\circ} \mathrm{C}$. Samples were stored at $4^{\circ} \mathrm{C}$ until flow cytometry analyses.

Statistical analysis. The results of RT-PCR were statistically analyzed using the one-way ANOVA test to determine the relationship of WNT5A mRNA expression mean values, respectively, among leukaemia cases, CR-cases, healthy cases and $\mathrm{CD} 34^{+}$cells. The results of MTT were statistically analyzed using the one-sample t-test. The results of WNT5A mRNA expression mean values of K562, U937, Jurkat before and after treated with Aza, respectively, were statistically 


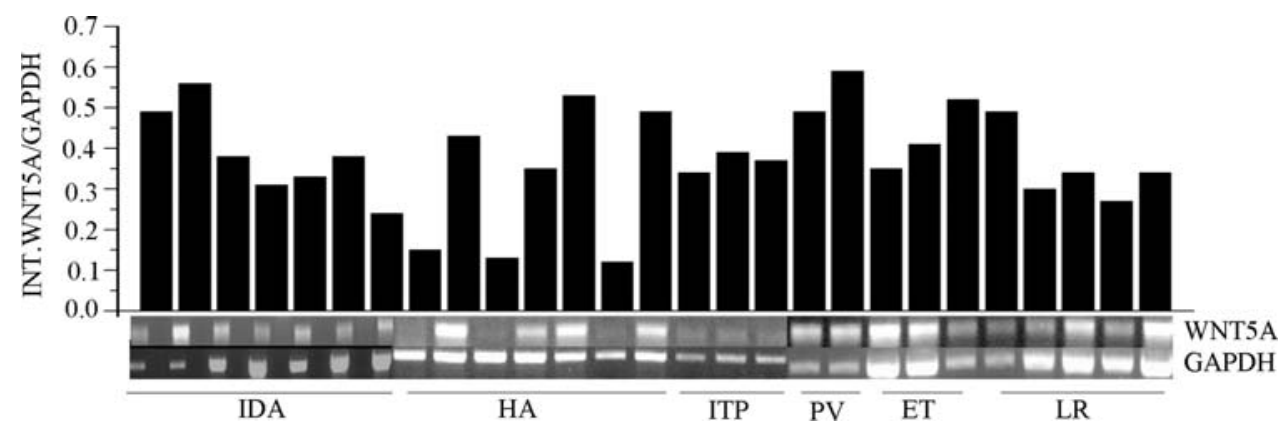

Figure 1. WNT5A expression in 27 non-malignant hematopoietic (NMH) cases. Total RNA was extracted from indicated samples and analyzed for WNT5A expression by RT-PCR. GAPDH expression was performed as a positive control. IDA, iron deficiency anemia; HA, hemolytic anemia; ITP, idiopathic thrombocytopenic purpura; ET, essential thrombocythemia; PV, polycythemia vera; LR, leukemoid reaction. The semi-quantitative mean value of WNT5A in NMH was $0.374 \pm 0.125$.

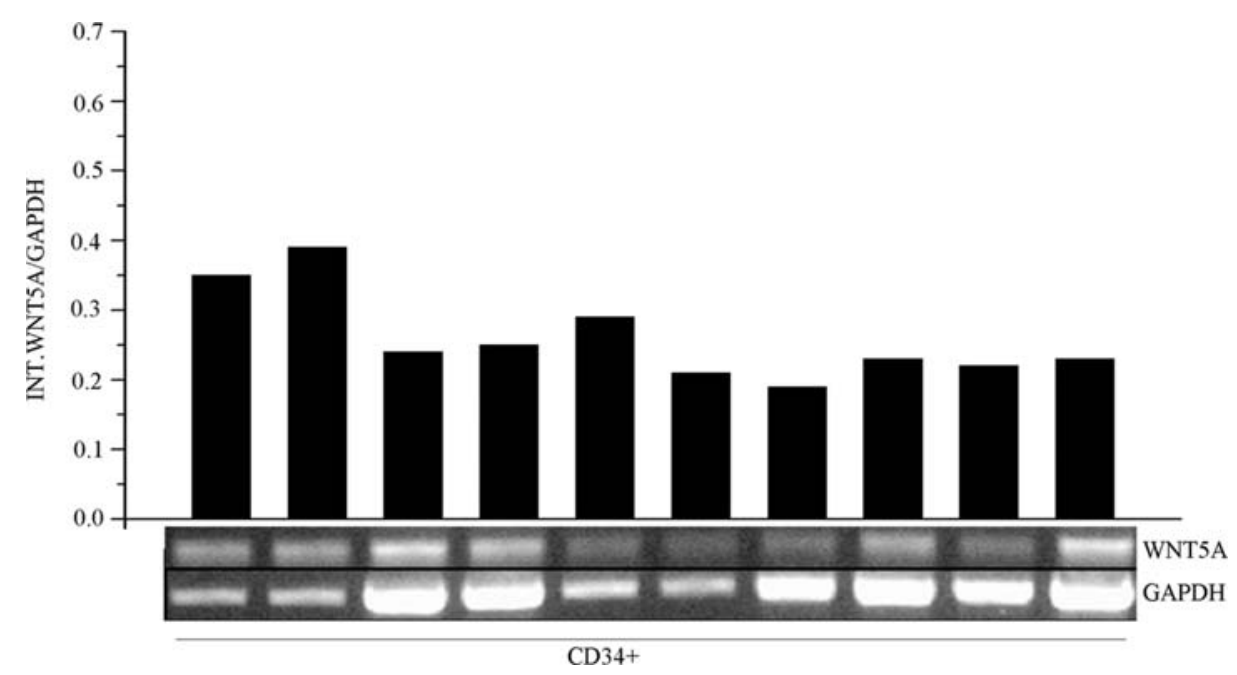

Figure 2. WNT5A expression in $10 \mathrm{CD} 34^{+}$cell. Total RNA was extracted from indicated samples and analyzed for WNT5A expression by RT-PCR. GAPDH expression was performed as a positive control. The semi-quantitative mean value of WNT5A in CD $34^{+}$cells was $0.260 \pm 0.064$.

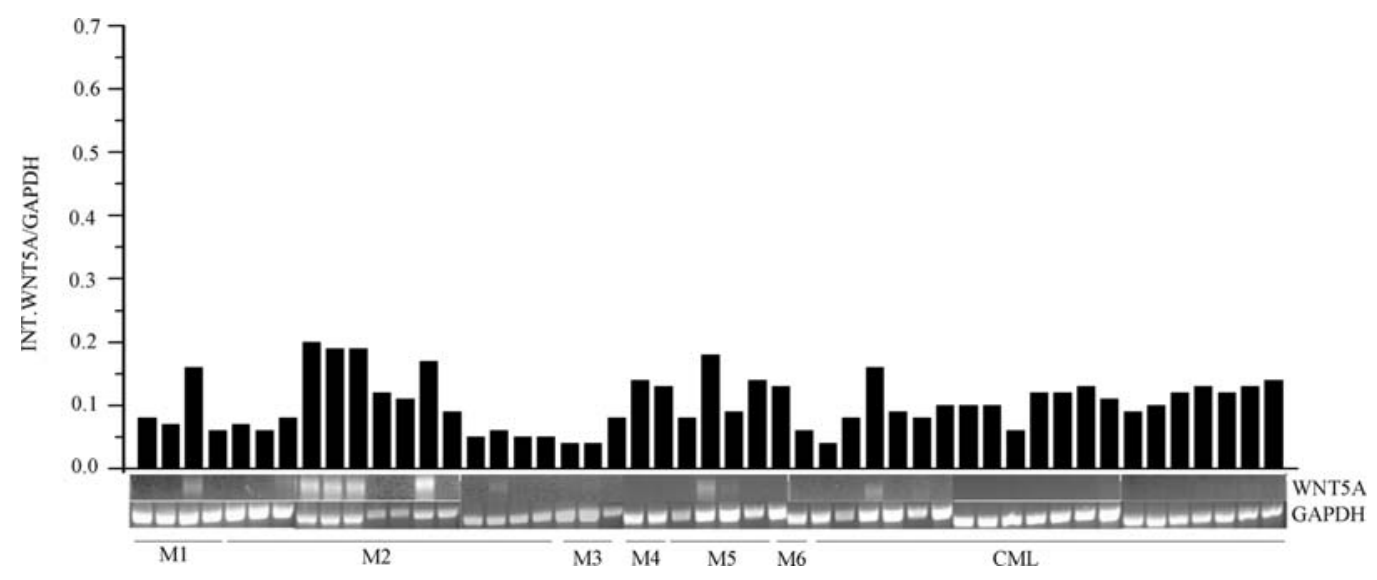

Figure 3. WNT5A expression in 49 myeloid leukaemia cases. Total RNA was extracted from indicated samples and analyzed for WNT5A expression by RT-PCR. GAPDH expression was performed as a positive control. The semi-quantitative mean value of $W N T 5 A$ in healthy cases was $0.105 \pm 0.042$.

analyzed using the Paired-samples t-test. The results of cell cycle distribution of K562 cell were analyzed using the $\chi^{2}$ test. SPSS 13.0 software was used. $\mathrm{P}<0.01$ was considered statistically significance.

\section{Results}

Expression of WNT5A mRNA. The semi-quantitative mean value $(\mathrm{x} \pm \mathrm{SD})$ of $W N T 5 A$ expression was $0.374 \pm 0.125$ in 


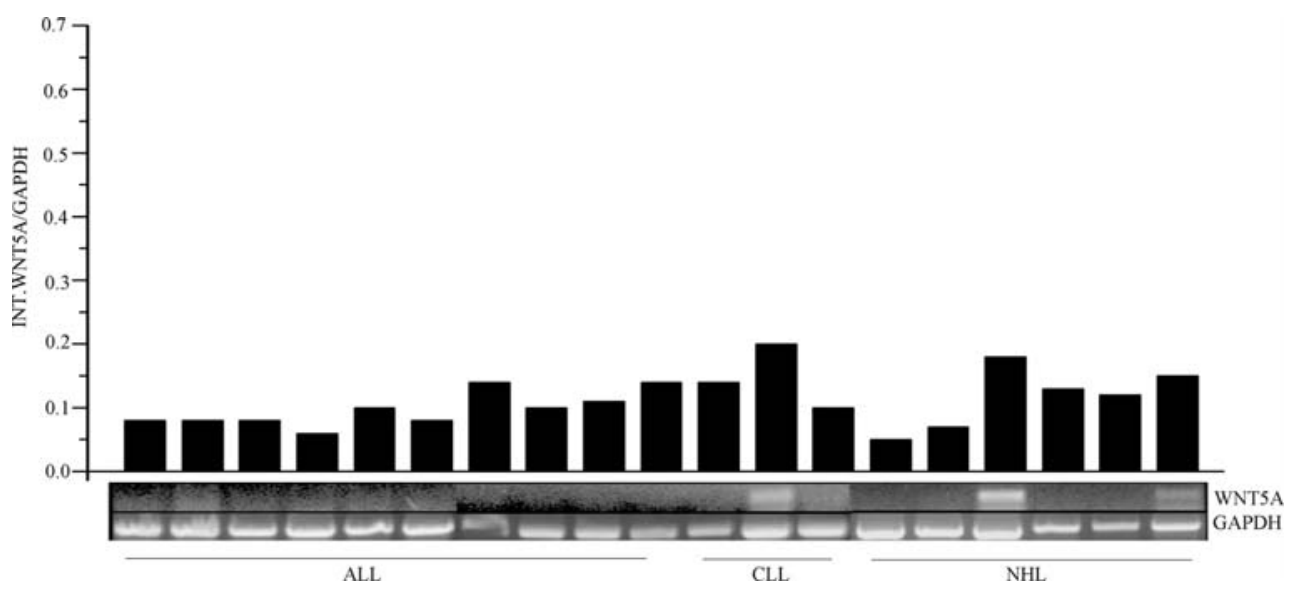

Figure 4. WNT5A expression in 19 lymphoid malignant tumor cases. Total RNA was extracted from indicated samples and analyzed for WNT5A expression by RT-PCR. GAPDH expression was performed as a positive control. The semi-quantitative mean value of WNT5A in healthy cases was $0.110 \pm 0.041$.

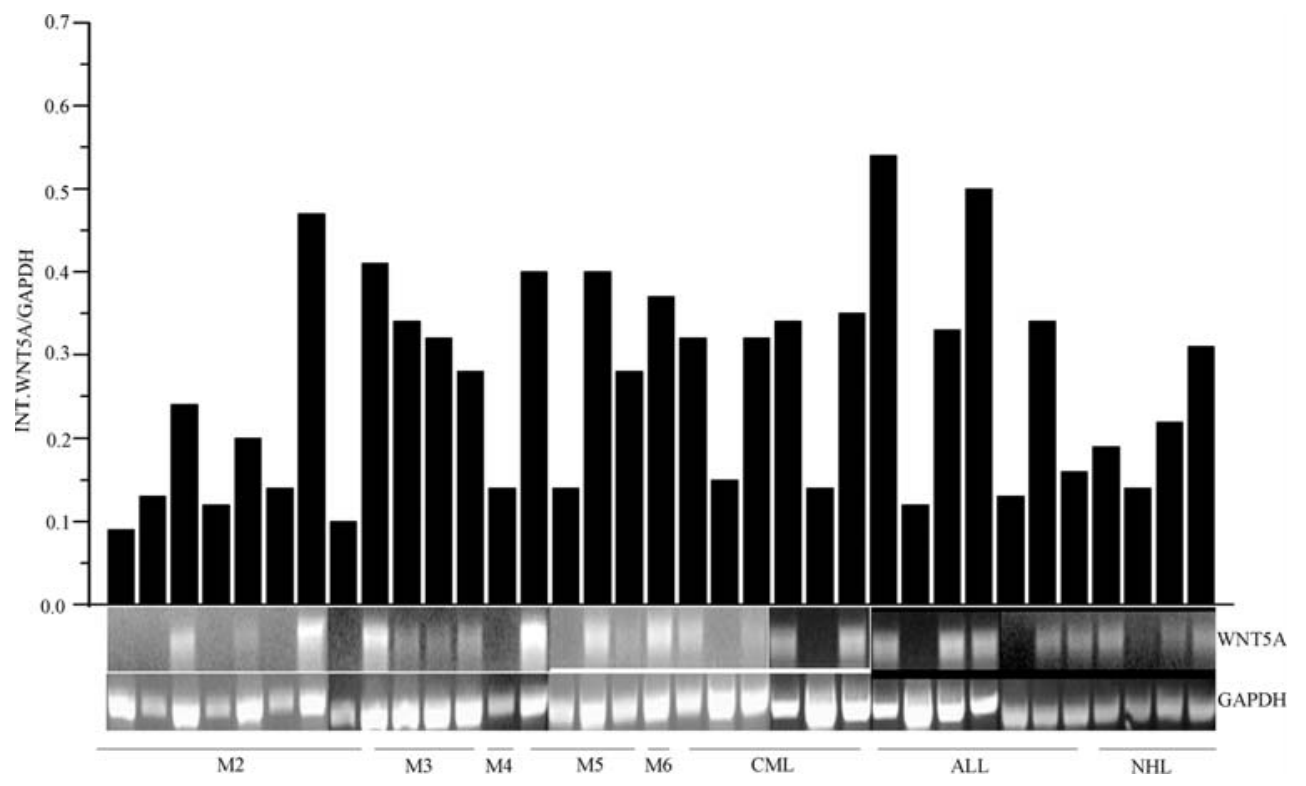

Figure 5. WNT5A expression in 35 leukaemia complete remissiom cases. Total RNA was extracted from indicated samples and analyzed for WNT5A expression by RT-PCR. GAPDH expression was performed as a positive control. The semi-quantitative mean value of WNT5A in healthy cases was $0.262 \pm 0.139$.

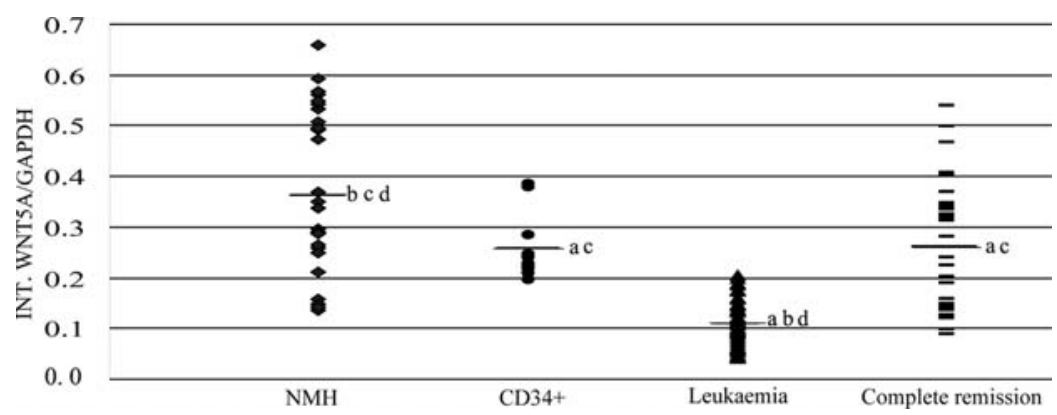

Figure 6. The semi-quantitative mean value (mean $\pm \mathrm{SD}$ ) of $W N T 5 A$ mRNA in NMH, CD34+ cells, leukaemia, complete remissiom cases. NMH, $0.374 \pm 0.125 ; \mathrm{CD}_{4}{ }^{+}, 0.260 \pm 0.064$; leukaemia, $0.106 \pm 0.042$; complete remissiom, $0.262 \pm 0.139$. a, $\mathrm{P}<0.01$, compared to $\mathrm{NMH} ; \mathrm{b}, \mathrm{P}<0.01$, compared to $\mathrm{CD} 34^{+} ; \mathrm{c}, \mathrm{P}<0.01$, compared to leukaemia; $\mathrm{d}, \mathrm{P}<0.01$, compared to complete remissiom.

healthy cases, $0.106 \pm 0.042$ in leukaemia cases, $0.262 \pm 0.139$ in CR-cases, $0.260 \pm 0.064$ in $\mathrm{CD} 34^{+}$cells, respectively. The expression level and the semi-quantitative mean value $(\mathrm{x} \pm \mathrm{SD})$ of WNT5A mRNA in all clinical cases and CD34+ cells are shown in Figs. 1-6. The expression of WNT5A mRNA was significantly lower in leukaemia cases than in 


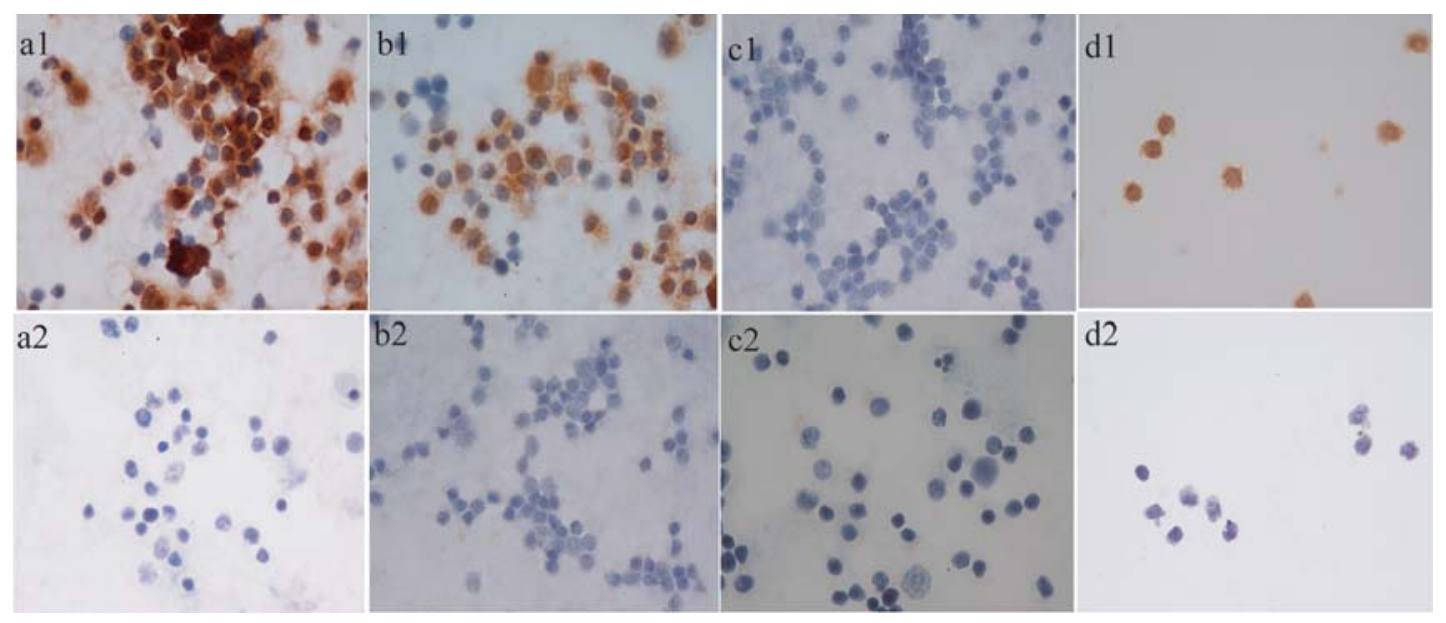

Figure 7. The expression of Wnt5a proten in NMH, CR, leukaemia, CD34+ by immunohistochemical analyses (x400). (a1) NMH; (b1) CR; (c1) leukaemia; (d1), CD34+; (a2, b2, c2 and d2) the correspondental negative control. Wnt5a protein was stained as light brown and mainly distributed in the cytoplasm. Wnt5a protein was positively expressed in $\mathrm{NMH}, \mathrm{CR}$ and $\mathrm{CD} 34^{+}$samples.

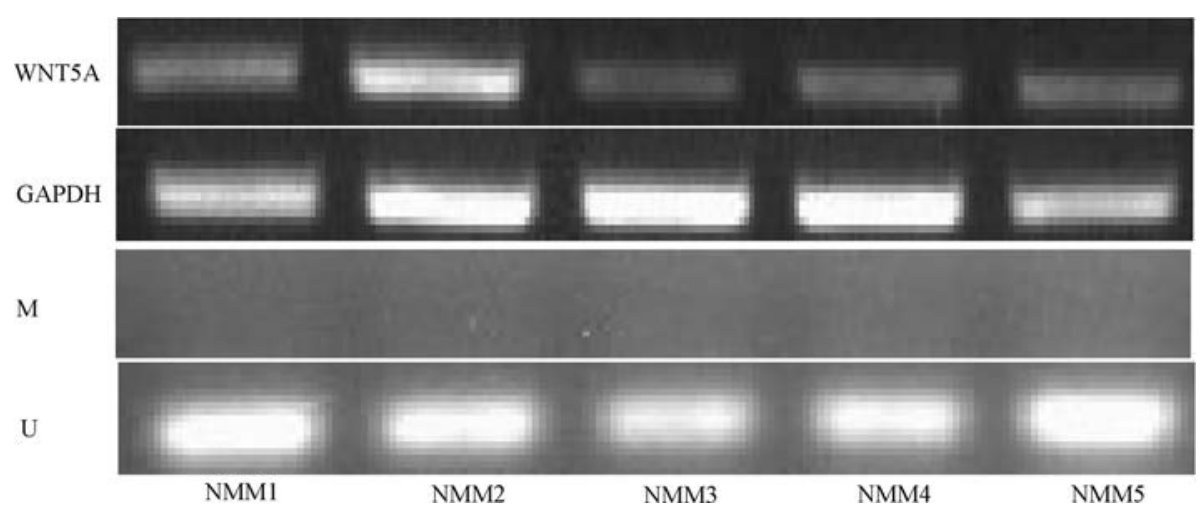

Figure 8. Expression and methylation analysis of WNT5A gene in non-malignant hematopoietic cases. NMH1-NMH5, the number of non-malignant hematopoietic cases. M, WNT5A methylated sequences of MSP analysis, U, WNT5A unmethylated sequences of MSP analysis. Positive expression and unmethylation status of the WNT5A gene was observed in all samples.

healthy cases $(\mathrm{P}=0.000)$, CR-cases $(\mathrm{P}=0.001)$ and $\mathrm{CD} 34^{+}$ cells $(\mathrm{P}=0.008)$. No significant difference was found among $\mathrm{CR}$-cases and $\mathrm{CD} 34^{+}$cells and healthy cases $(\mathrm{P}>0.01)$.

Immunocytochemistry staining of Wnt5a. The immunocytochemistry staining of Wnt5a was positive in healthy cases, $\mathrm{CD}^{+} 4^{+}$cells and CR-cases but was negative in leukaemia cases. All results of Wnt5a immunocytochemistry staining are shown in Fig. 7.

Expression of WNT5A gene in non-malignant hematopoietic cases and corresponding status of their promoter methylation. The expression of WNT5A transcripts was assessed by semi-quantitative RT-PCR in 27 non-malignant hematopoietic cases previously. Because the epigenetic events are important regulating mechanisms in gene transcripts, we decided to study the methylation status of the WNT5A promoter. By MSP, the WNT5A promoter was revealed to be unmethylated in non-malignant hematopoietic cases $(\mathrm{NMH})$ in accordance with the positive WNT5A expression. The results are shown in Fig. 8.
WNT5A promoter methylation in leukaemia patients is associated with underexpression of WNT5A transcripts. Methylation of WNT5A promoter was observed in $69 \%$ (47/68) samples amongst all the leukaemia patients, and only in $8.6 \%(3 / 35)$ amongst all the leukaemia CR patients (data not show). In order to further study the relationship between methylation and WNT5A expression, we show MSP and expression analysis of WNT5A gene in 12 leukaemia cases before and after their complete remission (CR). Amongst 12 leukaemia cases, methylation of WNT5A promoter was observed in $75 \%(9 / 12)$ samples, the positive rate of WNT5A mRNA expression was $25 \%$ (3/12). In contrast with complete remission cases, methylation of WNT5A promoter was observed in $8.3 \%(1 / 12)$ samples, the positive rate of WNT5A mRNA expression was $91.7 \%$ (11/12), only one complete remission case was found methylated of WNT5A promoter. The results are shown in Fig. 9.

Expression of WNT5A gene in leukaemia-derived cell lines is regulated by promoter methylation. In order to confirm the results of the MSP in NMH and leukaemia cases, we assayed 


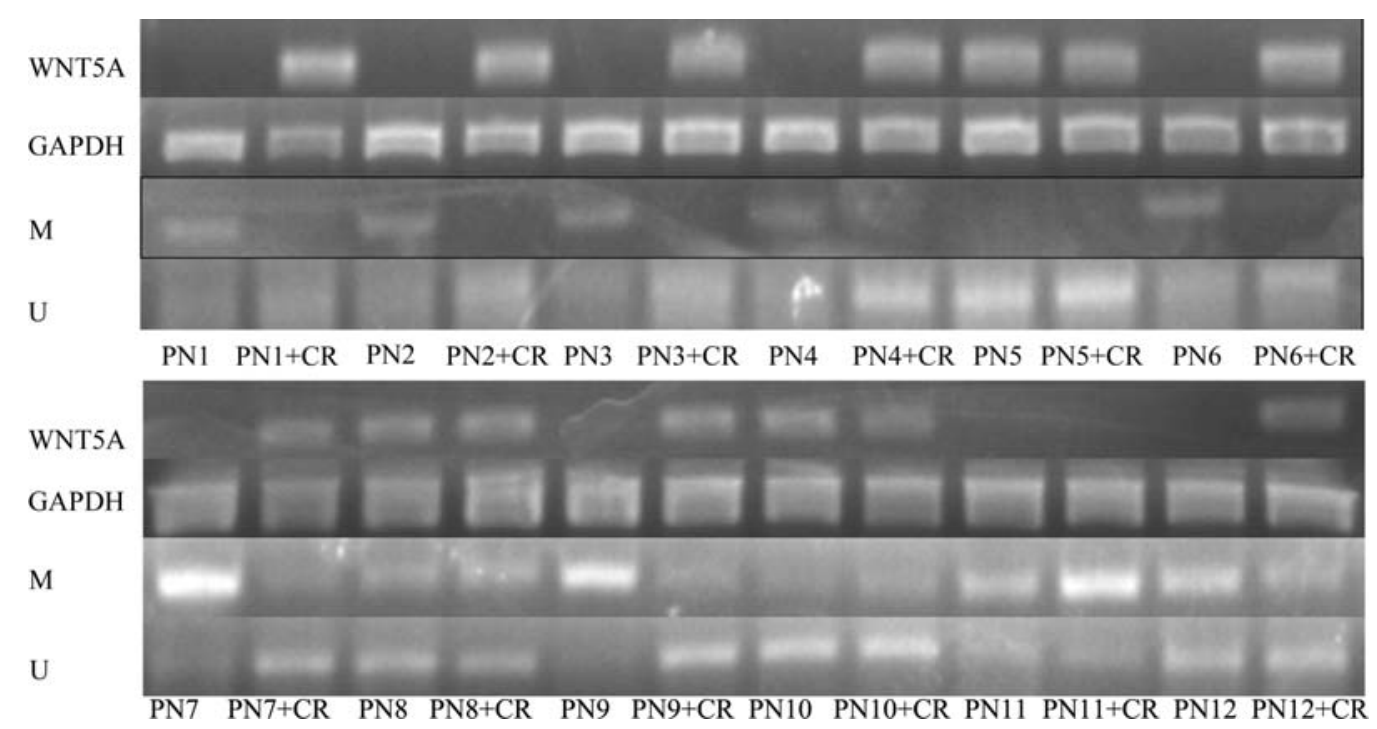

Figure 9. Expression and methylation analysis of the WNT5A gene in 12 leukaemia cases before and after their complete remission. PN1-PN12, patient number of leukaemia cases; PN1+CR-PN12+CR; patient number of complete remission leukaemia cases, correspondingly. M, WNT5A methylated sequences of MSP analysis; U, WNT5A unmethylated sequences of MSP analysis. Positive expression and unmethylation status of the WNT5A gene was observed in $\mathrm{PN}+\mathrm{CR}$ samples except in PN11+CR sample, and not in PN samples except in PN5, PN8 and PN10 samples.

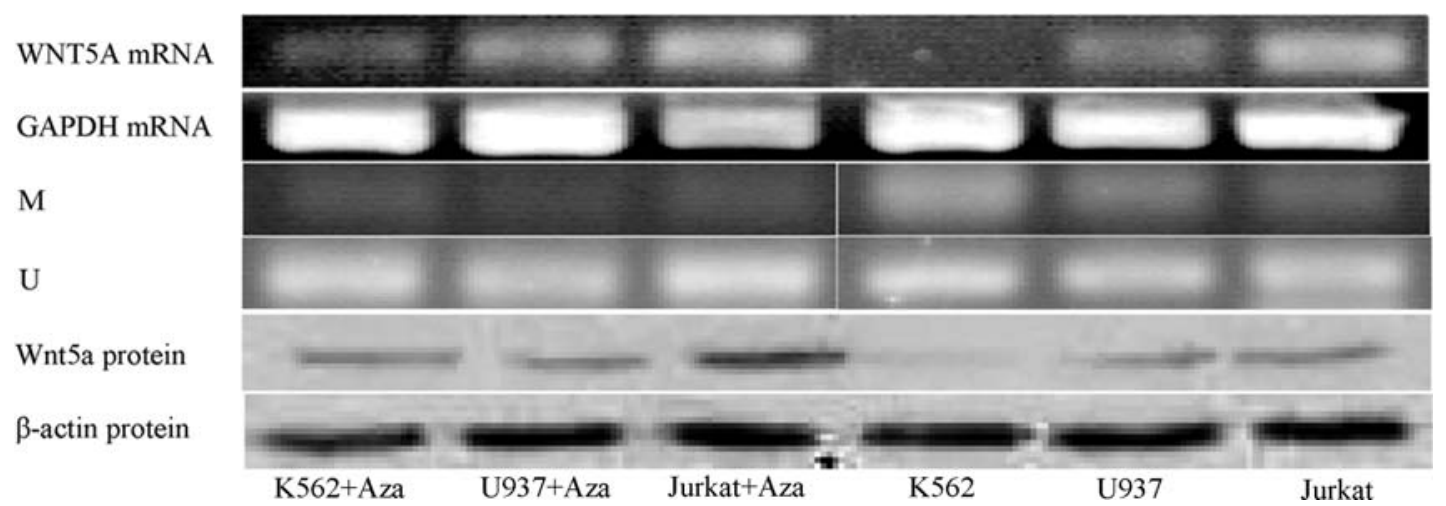

Figure 10. MSP and expression analysis of WNT5A gene in leukaemia cell lines before and after treatment with 5-Aza-20-deoxycytidine (Aza). K562+Aza, U937+Aza, Jurkat+Aza: K562, U937 and Jurkat was treated with Aza for 48 h. M, WNT5A methylated electrophoretic bands of MSP analysis; U, WNT5A unmethylated electrophoretic bands of MSP analysis. GAPDH mRNA, control for WNT5A mRNA. B-actin protein, control for Wnt5a protein. The semiquantitative mean value of WNT5A mRNA in K562, U937, Jurkat was $0.102 \pm 0.039,0.146 \pm 0.067,0.278 \pm 0.143$, respectively, and in K562+Aza, U937+Aza, Jurkat+Aza was $0.143 \pm 0.057,0.208 \pm 0.083,0.403 \pm 0.187$, respectively. The expression of WNT5A mRNA was up-regulated in K562+Aza, U937+Aza and Jurkat+Aza in comparison with K562, U937 and Jurkat, respectively ( $\mathrm{P}<0.01)$. The degree of WNT5A promoter methylation was extenuated in K562+Aza, U937+Aza and Jurkat+Aza contrasted to K562, U937 and Jurkat. The levels of Wnt5a protein expression in K562, U937 and Jurkat were up-regulated after the treatment with Aza.

the MSP and expression analysis of WNT5A gene in three leukaemia-derived cell lines (K562, U937 and Jurkat) before and after treatment with 5-Aza-2'-deoxycytidine (Aza). The expression of WNT5A was up-regulated after treatment with Aza for $48 \mathrm{~h}$ compared with the untreated controls, owing to the extenuated degree of WNT5A promoter methylation. Results were always confirmed by repeat MSP, RT-PCR and Western blot assay. The results are shown in Fig. 10.

Cytotoxic effect of Aza on $\mathrm{K} 562$ cells. Cells were treated with Aza on the concentration of $4 \mu \mathrm{M}$ at various times. Cell viability was determined by MTT assay. Treatment of Aza decreased the viability of K562 cells in a time-dependent manner. After 48-h treatment with Aza, the viability of K562 cell was significantly decreased. The results are shown in Fig. 11.

Treatment of K562 cells with Aza leads to cell cycle arrest. After 48-h treatment with Aza, K562 cells from different groups were collected and their cell cycle distribution was analyzed. These results suggest that demethylating agent Aza can induce K562 cell cycle arrest at G2/M phase. The results are shown in Fig. 12.

\section{Discussion}

Various WNT family members play important roles in haematogenesis by controlling the proliferation of progenitor 


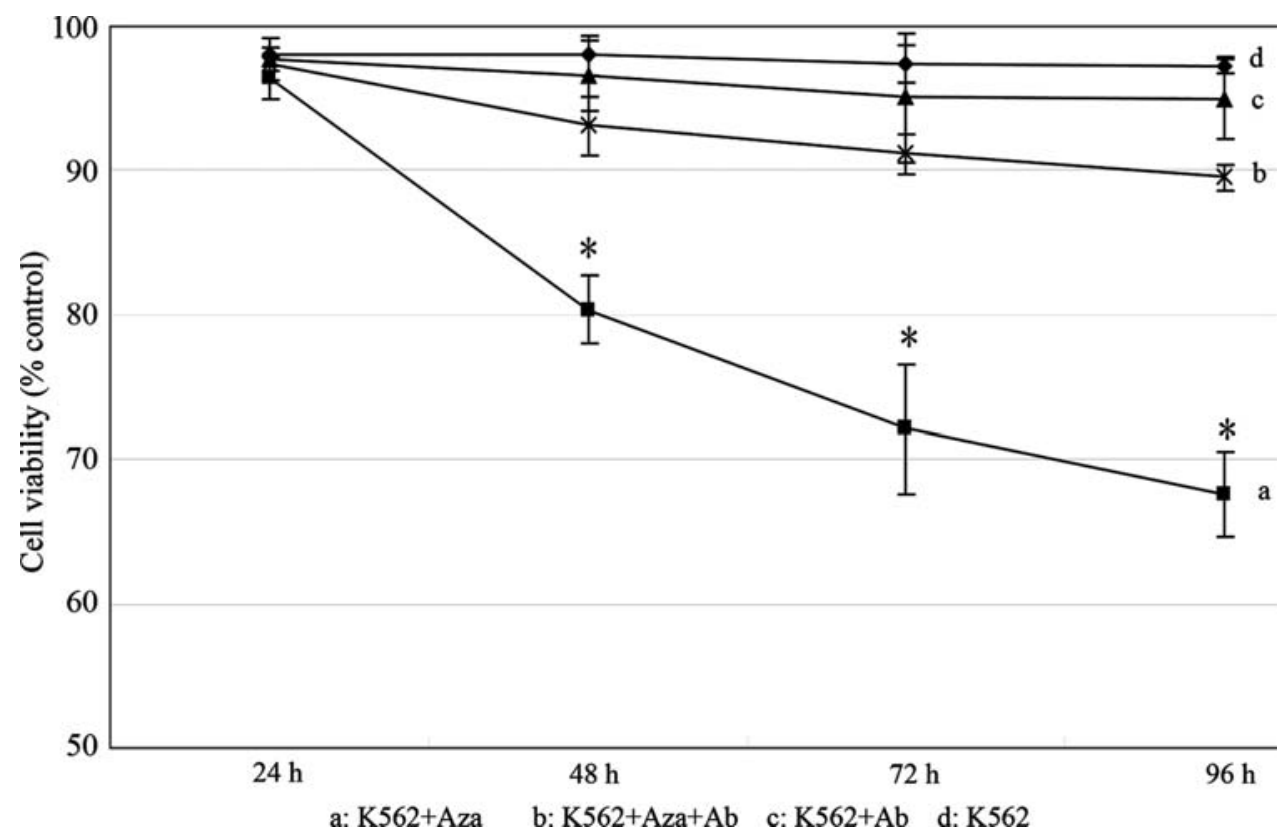

Figure 11. MTT assay was used to determine viability of K562 cells treated with Aza. Results represent the mean \pm SD of five experiments. The cell viability was calculated as: (OD Aza group-OD blank control)/(OD normal medium group-OD blank control) x 100\%. Normal medium group, K562 cells; K562+Aza, K562 cells treated with Aza; K562+Aza+Ab, K562 cells treated with Aza and polyclonal antibody of Wnt5a; K562+Ab, K562 cells treated with polyclonal antibody of Wnt5a. The cell viability were significantly lower at 48, 72, and $96 \mathrm{~h}$ in group of the K562+Aza compared to the normal medium group (P<0.01), but not in the groups of K562+Aza+Ab and K562+Ab.

A

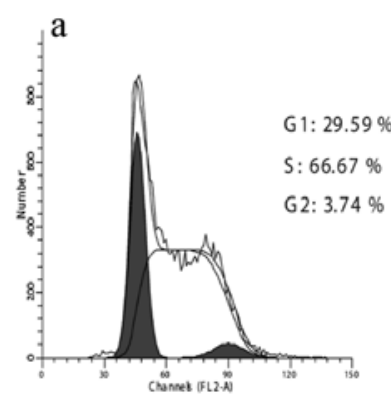

B

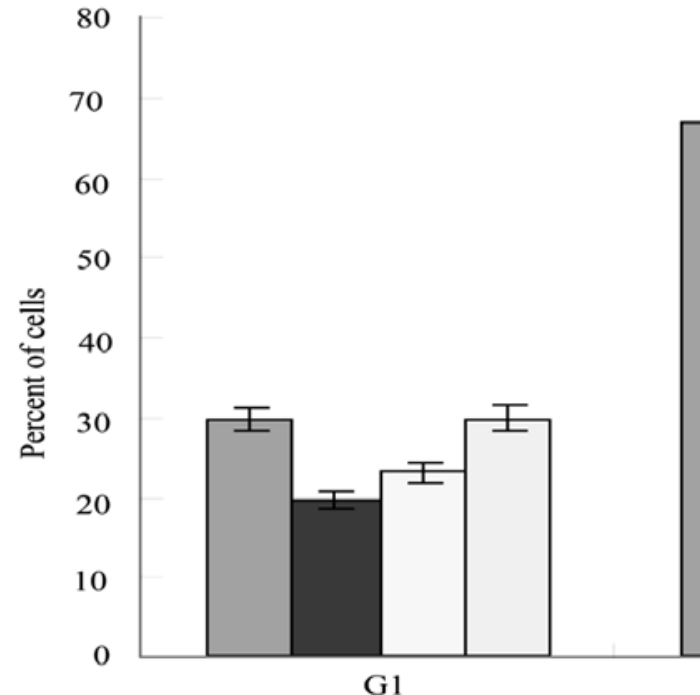

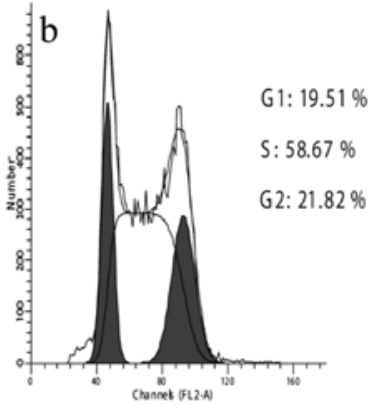
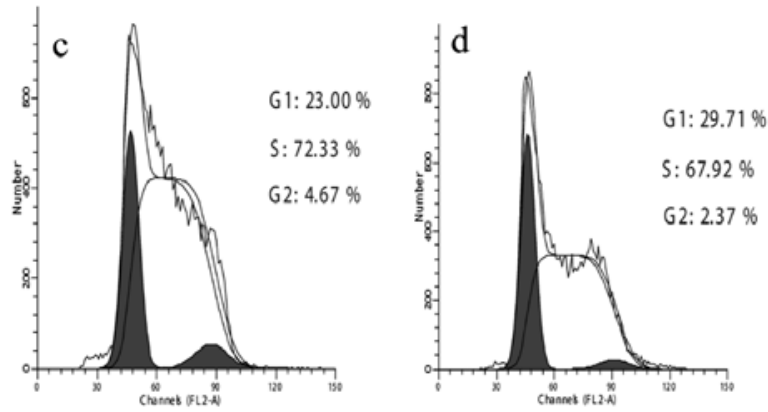

K562

K562+Aza

$\square \mathrm{K} 562+\mathrm{Aza}+\mathrm{Ab}$

$\mathrm{K} 562+\mathrm{Ab}$

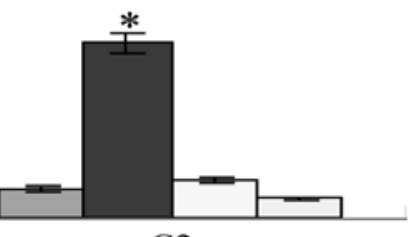

Figure 12. Flow cytometry analyses of the cell cycle distribution of K562 cells treated with Aza. K562+Aza, K562 cells treated with Aza; K562+Aza+Ab, K562 cells treated with Aza and polyclonal antibody of Wnt5a; K562+Ab, K562 cells treated with polyclonal antibody of Wnt5a. (A) Flow cytometry analyses cell cycle distributions of various K562 groups. (a) K562; (b) K562+Aza; (c) K562+Aza+Ab; (d) K562+Ab. (B) results of various K562 groups cell cycle distributions. The results are presented as the mean \pm SD of three experiments. The number of cells $(21.82 \%)$ in the G2/M phase was significantly increased in the group of K562+Aza $(\mathrm{P}<0.01)$ compared with the groups of K562, K562+Aza+Ab and K562+Ab. 
cells and might also affect the cell-fate decisions of stem cells. Therefore, WNT signaling is subject to strict molecular control, and dysregulated WNT signaling is implicated in the development of haematological malignancies. Previous studies have shown that reduction or absence of WNT5A expression is observed in some of the human primary leukaemia samples $(23,24-26)$. In this study, we describe for the first time that the levels of WNT5A expression in different kinds of nonmalignant hematopoietic cases (NMH), leukaemia cases, leukaemia CR cases, leukemic cell lines and CD34+ from umbilical cord blood. We also assayed the methylation status of WNT5A promoter in NMH, leukaemia, and leukaemia CR. We compared the level of WNT5A expression and the methylation status of WNT5A promoter in these cases before and after complete remission, respectively.

The results showed that WNT5A expression was downregulated in leukaemia cases due to the methylation of its promoter and was up-regulated in NMH and CR cases due to the demethylation of their promoter. The results indicated that the level of WNT5A expression was correlated with the methylation status of its promoter in leukaemia cases. The relationship between methylation and expression of WNT5A was further explored by the fact that when the leukemic cell lines K562, U937 and Jurkat were treated with Aza (a demethylating agent, which can induce demethylation of the WNT5A promoter region), up-regulation of WNT5A mRNA was observed. It was confirmed that the WNT5A expression was epigenetically regulated by methylation status of its promoter. In order to elucidate the relationship between the leukemic cell proliferation and up-regulation of WNT5A expression, K562 cell proliferation was studied by MTT assay and flow cytometry after treatment with Aza. Treatment of Aza decreased the viability of K562 cell in a timedependent manner. After 48-h treatment with Aza, the viability of K562 cell was significantly decreased, and the demethylating agent can induce K562 cell cycle arrest at $\mathrm{G} 2 / \mathrm{M}$ phase. These results suggest that Aza can induce demethylation of the WNT5A promoter, which can upregulate the $W N T 5 A$ expression. The up-regulation of Wnt5a level inhibits the K562 cell malignant proliferation, and arrests the K562 cell cycle at G2/M phase. Our findings suggest that Wnt5a can act as a suppressor factor in leukemogenesis. The conclusion is also supported by the fact that Wnt5a expression is resumed in leukaemia CR cases.

Román-Gómez et al reported that expression of the Wnt inhibitors sFRP1, sFRP2, sFRP4, sFRP5, WIF1, Dkk3, and Hdpr1 was down-regulated due to abnormal promoter methylation in ALL cell lines and samples from patients with ALL (27). Román-Gómez et al (27), Ying et al (25) and Martín et al (26) also reported that the expression of WNT5A can be down-regulated due to abnormal promoter methylation in leukaemic cell lines or leukaemia. In the present study, K562 cell proliferation was inhibited after treatment with Aza. It is possible that both the up-regulation of WNT5A and the Wnt inhibitors can inhibit the K562 cell proliferation through inhibiting the canonical Wnt pathway. To determine which is the case, we applied the polyclonal antibody of WNT5A to the K562 cells treated with Aza as a control. We found that the cell viability was significantly lower at 48,72, and $96 \mathrm{~h}$ after the treatment of Aza in the group of $\mathrm{K} 562+\mathrm{Aza}(\mathrm{P}<0.01)$, but was not significantly different in the groups of $\mathrm{K} 562+\mathrm{Aza}+\mathrm{Ab}$ or $\mathrm{K} 562+\mathrm{Ab}$, compared to the normal medium group. The cell cycle was arrested at $\mathrm{G} 2 / \mathrm{M}$ phase at $48 \mathrm{~h}$ after treated by Aza in group of the K562+Aza, but not in the groups of normal medium, K562+Aza+Ab and K562+Ab. The results confirmed that Wnt5a can inhibit the K562 cell malignant proliferation. Further study is needed to investigate the mechanism that cause the K562 cell cycle arrest at G2/M phase after treatment with Aza.

In addition, our research also showed that the WNT5A expression level of $\mathrm{CD}_{3} 4^{+}$cell was higher than that of leukaemia cases, but lower than that of NML. The level of WNT5A expression in $\mathrm{CD}_{3} 4^{+}$may be related to maintaining the hematopoietic progenitor cells moderate proliferation potential. The hematopoietic progenitor cells are responsible for the continuous production of blood cells. Thus, the proliferation potential is higher than that of the mature blood cells, but lower than that of the malignant proliferation in leukaemic cells. The level of WNT5A expression (higher than that of malignant proliferation leukaemic cells but lower than that of non-malignant proliferation cell of NML cases) may be one of the mechanisms which adjust the moderate proliferation potential of hematopoietic progenitor cells.

Currently, leukaemia diagnosis, treatment as well as prognosis evaluation are mainly based on the morphology analysis performed in the clinical laboratory. There are limitations for the morphology diagnosis. First, it can not be used for early diagnosis, since the morphological changes always lag the molecular alterations, and it generally requires more than $10^{6}$ malignant cells for reliable detection. Second, the accuracy of the diagnosis is compromised due to the objectivity of the method itself, as well as the complexity of cell morphology resulting from variations among individuals. In China, for example, according to the incomplete statistics, the diagnosis accuracy through morphological observation of patient bone marrow cells is only $70 \%$.

Thus, additional diagnostic methods are needed in order to improve the diagnostic accuracy. Molecular biomarkers have become promising diagnostic tools. For example, the measure of fusion gene expression is used in diagnosis of leukaemia or predicting its relapse. It is well established that measuring of fusion gene expression of BCR-ABL, PMLRAR $\alpha$, and AML-ETO is used in the diagnosis of certain CML, APL and AML, respectively. However, since these fusion genes are limited to certain types of leukaemia, a broad spectrum diagnosis marker and the index of curative effect assessment is needed that can diagnose leukaemia at an early stage reflecting the change required in treatment. Both our research and past reports have showed that there are remarkable differences in Wnt5a expression levels not only between normal and solid tumor tissues (16-19), but also between normal or non-malignant hematopoietic tissue and most blood malignancies (20-22,24,28). Loss of expression of Wnt5a may be one of the reasons that hematopoietic cells obtain malignant proliferation ability. Therefore, the complete remission leukaemia and non-malignant hematopoietic cases may be distinguished from the human primary leukaemia by the expression level of WNT5A, the level of $W N T 5 A$ expression can reflect the effect of treatment. We 
propose that WNT5A can act as a suppressor factor in leukemogenesis and can be used as a potential marker for curative effect assessment in leukaemia.

\section{Acknowledgements}

This study was supported by the National Natural Science Foundation of China (30973386), and the Major Program of Natural Science Foundation of Chongqing (CSTC2009BA5057), China. We are grateful to the Xin-Qiao Hospital, South-West Hospital of the Third Military Medical University China and to the donators for their bone marrow. Special thanks to Dr Tong Chuan He for his expert technical assistance.

\section{References}

1. Wodarz A and Nusse R: Mechanisms of Wnt signaling in development. Annu Rev Cell Dev Biol 14: 59-88, 1998.

2. Miller JR: The Wnts. Genome Biol 3: Reviews3001, 2002.

3. Logan CY and Nusse R: The Wnt signaling pathway in development and disease. Annu Rev Cell Dev Biol 20: 781-810, 2004.

4. Moon RT, Kohn AD, De Ferrari GV and Kaykas A: WNT and beta-catenin signalling: diseases and therapies. Nat Rev Genet 5: 691-701, 2004.

5. Kestler HA and Kuhl M: From individual Wnt pathways towards a Wnt signalling network. Philos Trans R Soc Lond B Biol Sci 363: 1333-1347, 2008.

6. Klaus A and Birchmeier W: Wnt signalling and its impact on development and cancer. Nat Rev Cancer 8: 387-398, 2008.

7. Clevers H: Wnt/beta-catenin signaling in development and disease. Cell 127: 469-480, 2006.

8. Reya T and Clevers H: Wnt signalling in stem cells and cancer. Nature 434: 843-850, 2005.

9. Jones $\mathrm{C}$ and Chen P: Planar cell polarity signaling in vertebrates. Bioessays 29: 120-132, 2007.

10. Kuhl M, Sheldahl LC, Park M, Miller JR and Moon RT: The Wnt/Ca ${ }^{2+}$ pathway: a new vertebrate Wnt signaling pathway takes shape. Trends Genet 16: 279-283, 2000.

11. Winkel A, Stricker S, Tylzanowski P, et al: Wnt-liganddependent interaction of TAK1 (TGF-beta-activated kinase-1) with the receptor tyrosine kinase Ror2 modulates canonical Wnt-signalling. Cell Signal 20: 2134-2144, 2008.

12. Ishitani T, Kishida S, Hyodo-Miura J, et al: The TAK1-NLK mitogen-activated protein kinase cascade functions in the Wnt$5 \mathrm{a} / \mathrm{Ca}(2+)$ pathway to antagonize $\mathrm{Wnt} /$ beta-catenin signaling. Mol Cell Biol 23: 131-139, 2003.

13. Torres MA, Yang-Snyder JA, Purcell SM, DeMarais AA, McGrew LL and Moon RT: Activities of the Wnt-1 class of secreted signaling factors are antagonized by the Wnt-5A class and by a dominant negative cadherin in early Xenopus development. J Cell Biol 133: 1123-1137, 1996.
14. Nomachi A, Nishita M, Inaba D, Enomoto M, Hamasaki M and Minami Y: Receptor tyrosine kinase Ror2 mediates Wnt5ainduced polarized cell migration by activating c-Jun N-terminal kinase via actin-binding protein filamin A. J Biol Chem 283: 27973-27981, 2008.

15. Mikels AJ and Nusse R: Purified Wnt5a protein activates or inhibits beta-catenin-TCF signaling depending on receptor context. Plos Biol 4: e115, 2006.

16. Saitoh T, Mine T and Katoh M: Frequent up-regulation of WNT5A mRNA in primary gastric cancer. Int J Mol Med 9: 515-519, 2002.

17. Jonsson M, Dejmek J, Bendahl PO and Bendahl PO: Loss of Wnt-5a protein is associated with early relapse in invasive ductal breast carcinomas. Cancer Res 62: 409-416, 2002.

18. Huang CL, Liu D and Nakano J: Wnt-5a expression is associated with the tumor proliferation and the stromal vascular endothelial growth factor-an expression in non-small-cell lung cancer. J Clin Oncol 23: 8765-8773, 2005.

19. Ripka S, Konig A, Buchholz M, et al: WNT5A, target of CUTL1 and potent modulator of tumor cell migration and invasion in pancreatic cancer. Carcinogenesis 28: 1178-1187, 2007.

20. O'Connell MP, French AD, Leotlela PD and Weeraratna AT: Assaying Wnt5A-mediated invasion in melanoma cells. Methods Mol Biol 468: 243-253, 2008.

21. Bui TD, Tortora G, Ciardiello F and Harris AL: Expression of Wnt5a is downregulated by extracellular matrix and mutated c-Ha-ras in the human mammary epithelial cell line MCF-10A. Biochem Biophys Res Commun 239: 911-917, 1997.

22. Kremenevskaja N, von Wasielewski R, Rao AS, Schofl C, Andersson T and Brabant G: Wnt-5a has tumor suppressor activity in thyroid carcinoma. Oncogene 24: 2144-2154, 2005.

23. Liang H, Chen Q, Coles AH, et al: Wnt5a inhibits B cell proliferation and functions as a tumor suppressor in hematopoietic tissue. Cancer Cell 4: 349-360, 2003.

24. Román-Gómez J, Jimenez-Velasco A, Cordeu L, et al: WNT5A, a putative tumour suppressor of lymphoid malignancies, is inactivated by aberrant methylation in acute lymphoblastic leukaemia. Eur J Cancer 43: 2736-2746, 2007.

25. Ying J, Li H, Chen YW, Srivastava G, Gao Z and Tao Q: WNT5A is epigenetically silenced in hematologic malignancies and inhibits leukaemia cell growth as a tumor suppressor. Blood 110: 4130-4132, 2007.

26. Martin V, Valencia A, Agirre X, et al: Epigenetic regulation of the non-canonical Wnt pathway in acute myeloid leukaemia. Cancer Science 101: 425-432, 2010.

27. Román-Gómez J, Cordeu L, Agirre X, et al: Epigenetic regulation of Wnt-signaling pathway in acute lymphoblastic leukaemia. Blood 109: 3462-3469, 2007.

28. Liang HL, Coles AH, Zhu ZQ, et al: Non-canonical Wnt signaling promotes apoptosis in thymocyte development. J Exp Med 204: 3077-3084, 2007. 